\title{
The Transition University-Work for Disabled People: A Needs Analysis
}

\section{Graziella D'Auria}

\author{
Master degree in Psychology, University G. d'Annunzio of Chieti - Pescara
}

Email: grazielladauria@yahoo.it

\section{Doi:10.5901/mjss.2016.v7n6p223}

\begin{abstract}
This paper analyses the influence of disability on education, training and career development of disabled people, by presenting a critical perspective on Italian legislation concerning inclusive policies. The starting point is a detailed historical review of the first interventions, along with a critical presentation of cross-cultural perspectives on school and work inclusion. The paper focuses on personal experiences of disabled people in entering the education system and the work contexts. From a methodological point of view the study adopted netnography to collect data from 30 disabled people. The main results suggest that individual characteristics play a central role in problems facing, with a rich spectrum of responses and call for new policies by the different stakeholders involved in inclusion.
\end{abstract}

Keywords: disability; education; job placement; inclusion; autonomy

\section{Introduzione}

Lo studio ha voluto esaminare l'impatto della disabilità nel percorso universitario e lavorativo della persona portatrice di handicap. Lo scopo della ricerca è stato quello di rilevare i vissuti provati da questi soggetti e sottolineare i bisogni che spesso non riescono ad essere percepibili all'occhio di chi non vive la condizione di svantaggio in prima persona.

Ripercorrendo alcune tappe storico-legislative relative all'integrazione del disabile a partire dal 1970 e da un confronto con la legislazione estera (come ad esempio le leggi statunitensi The Carl D. Perkins Vocational Applied Technology Education Act I-III, 1990, 1994, in IRSO, 2000 e le leggi svedesi: log um grunnskòla, 2008; log um framhaldsskòla, 2008), emerge l'avanguardia italiana sull'emanazione delle leggi, malgrado, spesso, sia tangibile l'arretratezza nell'applicazione pratica delle stesse. Riferendosi, ad esempio, all'evoluzione nella storia delle rappresentazioni sociali del disabile, si osserva uno scarso risultato nel superamento della visione negativa e limitata nei suoi confronti. Prevale ancora l'atteggiamento di pietismo, che considera l'inabile come un oggetto fallato, con la conseguenza che "il mondo normale" non riesce a stabilire un contatto proficuo con la persona in difficoltà. Anche quando si parla del disabile come un supereroe, in realtà lo si considera come qualcuno che ha compiuto delle azioni supreme, che vanno al di là delle sue potenzialità, senza prendere in considerazione che le possiede realmente. Quest'inquadramento di pensiero si riscontra anche nei clinici e negli esperti in generale, i quali rischiano di cadere nella trappola di inserire l'inabile in una categoria diagnostica senza considerare la persona in toto.

La limitatezza nella percezione delle reali potenzialità può generare una non soddisfacente crescita nell'autonomia e nell'autodeterminazione del disabile, fino ad impedirgli il raggiungimento di una posizione lavorativa che lo gratifichi, offrendogli una giusta realizzazione e che si integri assieme agli altri aspetti della vita. II lavoro dovrebbe essere, invece, un trampolino di lancio per il raggiungimento della massima soddisfazione in ogni settore. Questo tema è stato fonte di interesse per molti studiosi. Ad esempio, già a partire dal 1980 Donald Super ha proposto un'idea del concetto di sviluppo di carriera come processo di continua e permanente implementazione del concetto di sé confrontato con la realtà (Super, 1980). Arulmani e Nag-Arulmani (2004) hanno implementato un modello utile per superare alcune difficoltà nell'integrare il lavoro all'interno di tutta l'esistenza. Tale modello propone di considerare tutti gli ecosistemi nella progettazione di un profilo professionale che garantisca la piena realizzazione del soggetto.

Dalla raccolta delle informazioni, riguardanti i progressi raggiunti in relazione alla risposta del bisogno integrativo del disabile in ambito formativo e professionale, emergono buoni risultati sia nel settore scolastico sia in quello universitario e/o lavorativo.

Nel settore scolastico, ad esempio, si è passati dal relegare l'istruzione dei disabili all'interno di istituti speciali, per giungere alla loro completa integrazione nella scuola pubblica (legge 517/1977). È rilevante l'effetto della legge 104/1992, che ribadisce ed amplia il principio dell'integrazione, con l'obiettivo di sviluppare le potenzialità nell'apprendimento, nella 
comunicazione e nella socializzazione, predisponendo il P.E.I. (Piano Educativo Individualizzato), anche se esso, spesso, tende al raggiungimento di obiettivi curriculari, trascurando altre competenze che lo studente potrebbe sviluppare e che sarebbero molto più utili nella professionalità futura (cfr. Ferrucci \& Cortini, 2015). Ulteriormente, l'interesse per la piena autorealizzazione del soggetto portatore di handicap è stato reso più vivo dalla legge 53/2003, la quale ha determinato l'obbligo di predisporre sussidi tecnici e didattici che favoriscano la piena autonomia nel conseguire i propri obiettivi individuali.

Nell'ambito universitario, il progresso più brillante ottenuto, è garantito dall'istituzione del Tutorato Specializzato grazie alla legge 17/1999. Viene riconosciuto, in tal modo, al Tutor il compito di orientare coloro che, per condizioni di ridotta autonomia dovuta a cause psicologiche, fisiche, sociali o culturali, necessitano di sostegno. II Tutorato Specializzato non ha il fine di proporre percorsi facilitati, ma di rilevare gli ostacoli e ricercare strade alternative. Viene valorizzato il potere relazionale e l'empatia assume un ruolo determinante nell'apprendimento. Nonostante questi traguardi positivi rimane ancora poco enfatizzata la visione ecologica, la quale potrebbe attivare la partecipazione e il miglioramento delle relazioni con il territorio e la comunità locale, per sviluppare la funzione di community care.

Nel 2007, con la Convenzione delle Nazioni Unite, si sviluppa un crescente interesse all'impatto positivo che l'impiego occupazionale ha sull'autostima e sulla realizzazione dei bisogni del soggetto svantaggiato. Essa ha l'obiettivo di superare la discriminazione e la considerazione del disabile come dotato di scarse risorse per il mondo lavorativo ed offrire un'opportunità di socialità, scambio e confronto. Si ha, quindi, una concezione dellindividuo non più come destinatario passivo dei servizi socio-sanitari, ma come persona consapevole della propria unicità e identità. L'Italia oltre a recepire in pieno questa direttiva, attua nel 2010 l'Osservatorio Nazionale sulla condizione delle persone con disabilità all'interno del contesto lavorativo. Esso predispone un programma biennale di promozione dei diritti, di integrazione sociale e di indagini finalizzate ad individuare aree prioritarie su cui intervenire. La legislazione nazionale ha provveduto a facilitare l'inserimento, delle persone disabili, nel mondo del lavoro attraverso la predisposizione di forme di sostegno e supporto. È stata fissata una quota di riserva, che offre la possibilità di assumerne un disabile ogni 15 dipendenti. Inoltre, è stato predisposto che il collocamento fosse mirato, mediato e condiviso, predisponendo il Piano di Intervento Personalizzato (P.I.P.), che tiene conto delle capacità lavorative residue del disabile e deve essere progettato con il datore di lavoro, considerando anche la rete sociale dei colleghi (legge 68/1999). Successivamente all'assunzione del candidato in azienda è opportuno monitorare alcuni elementi per rendere agevole l'integrazione, come assicurare il contatto tra lavoratore, tutor, colleghi e superiori, creare le condizioni organizzative e/o logistiche ottimali e fornire ausili che migliorino l'accoglienza (legge 196/1997). Sfortunatamente, nonostante gli sforzi per rispondere alle varie necessità dei soggetti con maggiori difficoltà, che vivono una più intensa esclusione sociale, restano ancora molti gli ostacoli per la loro completa integrazione.

I risultati positivi che sono stati raggiunti devono essere potenziati in modo da rispondere a quelle richieste rimaste sommerse. Spesso gli interventi sono settorializzati ed impediscono di osservare la persona nel suo complesso. Inoltre, ci si dovrebbe orientare verso una maggiore considerazione delle differenze. Queste andrebbero vagliate come fonte di stimoli e ricchezze, di cui tutta la comunità può avvantaggiarsi se attentamente valutate.

II presente lavoro ha voluto offrire un contributo al raggiungimento di un tale risultato. L'analisi delle parole dei diretti interessati ha portato in superficie ulteriori bisogni o necessità, indici di quelle differenze che andrebbero ascoltate.

\section{Materiali e Metodi}

L'analisi è stata condotta su un campione di 30 soggetti, di cui 11 maschi e 19 donne, con un grado di disabilità che varia da lieve a grave. Nello specifico, 9 affetti da Tetraparesi Spastica, di cui 2 con Disturbo del Linguaggio; 5 Paraplegici; 2 Sordi; 3 Non Vedenti; 2 Ipovedenti, di cui uno con Deficit Ipofisario Multiplo; 1 con Displasia che causa diverse compromissioni; 1 con Sindrome di Down; 2 con Distrofia Muscolare; 1 con Atrofia Muscolare Spinale; 1 con Atassia e 1 con Epilessia.

La ricerca è stata condotta seguendo un approccio qualitativo, in quanto non vi era un'ipotesi iniziale da validare e, dunque, la possibilità di adottare una logica nomotetica. In dettaglio è stata condotta un'analisi delle testimonianze rilasciate dai soggetti.

La ricerca qualitativa è definita come "quel tipo di ricerca che adotta un approccio naturalistico verso il suo oggetto di indagine, studiando i fenomeni nei loro contesti naturali, tentando di dare loro un senso, o di interpretarli, nei termini del significato che la gente dà ad essi" (Denzin \& Lincoln, 1994, pp.7-8 Cicognani, 2010). Per analizzare i dati essa impiega una vasta gamma di metodologie e tecniche, le quali possono essere utilizzate singolarmente 0 contemporaneamente, sia in modo simultaneo che sequenziale, in un ottica di triangolazione metodologica (Cortini, 2014). La ricerca qualitativa è caratterizzata da una prospettiva di unicità del fenomeno indagato, che viene considerato nella sua globalità e 
complessità; in tale metodo di ricerca l'oggetto di studio sono gli individui che interagiscono tra loro generando linguaggi condivisi e modificabili. L'obiettivo principale della ricerca qualitativa è quello di rilevare fenomeni sociali nuovi o poco conosciuti o per i quali le teorie esistenti sono incomplete e non riescono a fornire un'interpretazione esaustiva. Indaga, quindi, le spiegazioni di un fenomeno senza preoccuparsi del livello di generalizzabilità, che risulta limitato a causa della specificità dell'oggetto di indagine. Inoltre, la rappresentatività del campione non è un aspetto prioritario, perché lo scopo dell'indagine qualitativa non è scoprire leggi generali. L'approccio qualitativo riconosce la peculiarità di ogni studioso, la sua appartenenza ad una determinata cultura, società, con credenze, valori ed interessi personali, che non esimono la ricerca dai punti di vista soggettivi. Per tale motivo i valori del ricercatore devono essere resi espliciti in quanto fattori determinanti. Tale paradigma, infatti, prevede un'influenza reciproca tra ricercatore e partecipanti all'indagine e considera fondamentale la capacità interpretativa e l'abilità di concettualizzazione dello studioso. Viene valorizzata la circolarità e l'interdipendenza tra le fasi di raccolta, analisi e teorizzazioni dei dati, che determina un basso livello di strutturazione e la possibilità di integrazione degli aspetti dell'indagine se necessario.

Tra le varie metodologie di cui si avvale la ricerca qualitativa si è optato per il reperimento delle testimonianze da sottoporre ad analisi e per l'utilizzo dell'intervista focalizzata. Questa ha lo scopo di esaminare le reazioni dei soggetti a materiale-stimolo. Le domande vengono poste in assenza di direttività, favorendo in tal modo la libera espressione del vissuto dei partecipanti e l'emergere del significato e dell'effetto che lo stimolo procura nel soggetto. Questa è sembrata la scelta più opportuna per riuscire a carpire le opinioni che i soggetti hanno sull'argomento formazione, lavoro ed università legata alla disabilità. La richiesta specifica, rivolta ai partecipanti, è stata quella di raccontare, in qualità di disabile, le proprie emozioni, gli atteggiamenti, le sensazioni e le opinioni riguardo al vissuto scolastico, universitario e lavorativo. La libertà di espressione ha favorito l'emergere di argomenti ed esperienze sul vissuto personale degli intervistati, che ha permesso l'indagine di aspetti ulteriori rispetto al focus sondato.

D'altra parte è da considerare che è stato necessario l'impiego di un'intervista esperta, chiamata da Marshall e Rossman (1995) anche d'élite o a persone chiave. Essa viene condotta su persone che hanno una certa esperienza in un dato settore 0 che rappresentano un gruppo. In questo caso la direttività è doverosa, perché la traccia viene pensata in modo da far intuire che anche il ricercatore ha una certa conoscenza sull'argomento di competenza. Infatti, nello studio sia i soggetti esaminati sia il ricercatore appartengono alla categoria dei disabili, dunque esperti della condizione. Ciò ha permesso una migliore intesa ed empatia che ha favorito il riconoscimento di segnali altrimenti non percepibili da chi non appartiene al gruppo.

L'analisi dei dati è stata condotta seguendo la logica qualitativa che, secondo Dey (1993) implica la frammentazione dei dati in unità più semplici e la loro ricomposizione in modi nuovi. Oltre alla descrizione dei dati è previsto un riferimento agli oggetti o eventi ai quali essi si riferiscono, allo scopo di comprenderli ed interpretarli, per poi utilizzarli eventualmente ai fini della previsione. L'analisi procede secondo un'ottica circolare e prevede la lettura ripetuta delle trascrizioni. II ricercatore deve investire tutte le sue capacità creative ed interpretative, le quali potranno condurre all'individuazione di ulteriori temi connessi a quelli già individuati. Nel corso del lavoro si riflette sulle possibili spiegazioni delle classificazioni in cui sono disposte le opinioni relative ad un dato argomento oppure si descrivono $i$ temi in base alla tipologia. Attraverso la categorizzazione si sviluppa la teoria oppure la definizione dell'atteggiamento dei soggetti in base alle loro risposte. Ė richiesta altresì, nell'analisi qualitativa, la concentrazione sulla complessità di un determinato argomento, al fine di scioglierne eventuali ambiguità e contraddizioni evidenziate dalle riflessioni di un partecipante. Nel lavoro, in effetti, sono state raccolte le testimonianze in un dataset e successivamente si è proceduto alla lettura ripetuta dei testi trascritti. Questo ha permesso l'individuazione di alcuni cluster, ai quali se ne sono aggiunti nuovi nelle successive riletture, effettuate per controllare la presenza di espressioni che confluissero nelle categorie individuate. Abbiamo preliminarmente effettuato un'analisi testuale dell'intero corpus dei dati, sottoponendolo al software T-Lab (Cortini \& Tria, 2014), per evidenziare le occorrenze e co-occorrenze di parola; abbiamo poi proseguito con un'analisi esclusivamente qualitativa.

Bisogna considerare che nella ricerca è stato opportuno anche l'utilizzo dell'analisi narrativa. Lieblich (1998) e collaboratori considerano tale qualunque studio che usa ed analizza materiale narrativo, il quale può essere scomposto rispetto ad una molteplicità di dimensioni. Le ipotesi non sono stabilite a priori, ma emergono nel corso della lettura del materiale raccolto, sempre in un processo circolare. L'analisi narrativa non richiede una replicabilità per la validazione, ma si affida alle capacità interpretative del ricercatore. Le diverse modalità di conduzione dell'analisi narrativa vengono classificate in base a due dimensioni. La prima comprende il versante olistico, che esamina la storia nella sua globalità e il versante categoriale che, invece, si concentra su sezioni o singole parole. Nella seconda dicotomia, invece, il contenuto si concentra, a volte, sull'aspetto esplicito in base al punto di vista del narratore ed, altre volte, su quello implicito, interrogandosi sul significato trasmesso dalla storia. La forma, viceversa, dà spazio alla struttura, alla sequenza degli eventi, ai sentimenti evocati dal racconto 0 alla complessità e alla coerenza con l'asse temporale. Quest'analisi si è 
ritenuta opportuna in quanto le testimonianze si sono rilevate vere e proprie biografie delle persone che hanno offerto il loro contributo. Pur non trattandosi di intere storie di vita è innegabile che le informazioni fornite riguardavano dei lassi temporali abbastanza ampi, che abbracciavano quindi una buona parte dell'esistenza dei soggetti. Analizzando i racconti dei partecipanti allo studio sono emersi molti bisogni ed esigenze non percepite dai legislatori e che non hanno trovato riscontro nelle azioni già intraprese a favore di un'adeguata integrazione scolastica, universitaria e lavorativa.

Nell'analizzare le testimonianze è stata utilizzata anche la metodologia dell'analisi metaforica. La tradizione aristotelica, nella Poetica (334-330 a.C.), descriveva la metafora come l'uso, in un dato contesto concettuale, di un

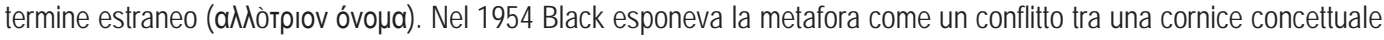
(frame) e un concetto estraneo (focus). A tali approcci si sono sostituite recenti prospettive che definiscono la metafora come sostituto (Fontanier, 1968; Genette, 1968; Groupe $\mu$, 1970), come estensione di significato di una parola (Dumarsais, 1988) e come fonte di concetti condivisi (Lakoff, Johnson, 1981; Lakoff, Turner, 1989). Tutte le teorie esistenti sulla metafora espongono il trasferimento di un concetto in un ambito diverso da quello originario. Se precedentemente veniva considerato un semplice accessorio linguistico, pian piano gli studiosi hanno cominciato a considerarlo un processo tipico della mente umana che permette di esplicitare il modo in cui la persona vede il mondo. L'espressione metaforica rappresenta un meccanismo di natura cognitiva che permette al nostro sistema concettuale di stabilire delle corrispondenze sistematiche di tipo analogico tra diversi domini semantici e/o di esperienza. Concettualizzando la realtà essa la incornicia in categorie attraverso la proiezione di una mappa cinestesica ad un livello cognitivo più astratto. Per una corretta analisi della metafora facciamo riferimento alle dimensioni sovraindividuale vs individuale che riflettono, rispettivamente la convenzionalità vs non convenzionalità del linguaggio metaforico considerato. Parliamo di dimensione sovraindividuale per intendere le competenze linguistiche metaforiche condivise da una specifica comunità linguistica. Viceversa, a livello individuale, la metafora è considerata in relazione all'uso che un individuo fa dei pattern metaforici, propri della comunità di appartenenza, per poi applicarli attraverso atti comunicativi in specifici contesti. Pertanto per convenzionalità intendiamo il grado in cui l'espressione metaforica è radicata e diffusa nel vocabolario della comunità e del parlante; mentre, la non convenzionalità denota l'uso originale con cui, in specifiche circostanze, viene applicato il linguaggio metaforico dal singolo individuo.

\section{Interpretazione delle Testimonianze}

Il lavoro di ricerca è iniziato con il campionamento dei soggetti da cui ricavare poi testimonianze e storie di vita, in particolare relative alla formazione scolastico/universitaria e all'ingresso nel mondo del lavoro. Fonte principale di individuazione e raccolta del materiale di analisi è stato il web. L'impiego di tale strumento è stato dettato dalla facilitazione nel reperimento dei soggetti che potevano fornire una testimonianza. Inoltre, attraverso la comunicazione via web le persone si sentono più libere di esprimere la loro condizione e sono meno soggette, quindi, ai pregiudizi che si possono presentare. Non meno importante risulta anche la maggiore agevolazione che scaturisce dall'uso del web per le persone disabili. In questo modo, infatti, è stato possibile per loro evitare uno sconfortevole spostamento fino al luogo designato per le eventuali interviste. II campione, di 30 soggetti, si compone di persone sconosciute e di alcuni contatti personali già esistenti. L'analisi delle testimonianze raccolte si è concentrata in particolare sulle tematiche e metafore prevalenti nelle ricostruzioni relative alla rappresentazione della disabilità, alla formazione scolastico-universitaria e al lavoro.

In seguito all'analisi è stato possibile rintracciare alcuni cluster costanti presentati da diversi soggetti. Tra quelli con valenza positiva troviamo: grinta-tenacia-determinazione; riscatto-rivincita-rivalsa; competenza-orgoglio; desiderio-forte volontà.

Molti di più purtroppo risultano quelli con valenza negativa: sfiducia-rassegnazione; delusione-amarezza; sconfitta-ripiego; rabbia-rancore; accusa; diversità-inadeguatezza-sentirsi di peso; fatica-sforzo.

Sono emersi anche dei temi verso i quali i soggetti hanno dimostrato una certa ambivalenza. Alcuni hanno manifestato una serena accettazione della propria diversità, altri, invece, hanno usato parole che lasciano intuire velatamente una non accettazione di essa. II concetto di lotta-sfida-difficoltà ha mostrato la stessa duplice valenza, così come si è verificata una certa contrapposizione nei confronti del cluster relativo alla Fede. Ė interessante osservare che molti dei soggetti hanno espresso le loro idee ed i loro propositi rispetto al tema dell'amore. Ciò sottolinea che, all'interno dei progetti di vita, questo aspetto può essere integrato con quello della formazione e del lavoro.

È esplicativo riportare integralmente la testimonianza fornita da una ragazza, nella quale si racchiudono molte tematiche espresse da tutti i partecipanti alla ricerca. Sicuramente i temi non sono tutti presenti in questo trafiletto ma, per ragioni di spazio, esso sintetizza molto bene gli argomenti ed inoltre rende l'idea del tipo di analisi che è stata svolta 
sul materiale.

"Sono Annamaria, una ragazza disabile, affetta da tetra paresi spastica, una patologia che mi accompagna dalla nascita, quando, a causa di un parto prematuro riporto danni neurologici che verranno diagnosticati a distanza di un anno dalla mia venuta al mondo.

E' da quel momento in poi che inizia la mia storia piena di grandi emozioni che, nell'alternarsi di momenti positivi e negativi, segna il mio percorso di crescita sia biologico che personale ed affettivo.

Dire di aver mosso i primi passi sarebbe solo una simpatica battuta, perché la mia disabilità non mi ha mai consentito di camminare da sola; è innegabile però, che vivere e sperimentare il limite ad una vita quotidiana di totale autonomia, mi ha insegnato molto!!!!

Grande importanza nella mia crescita ha avuto la famiglia, in quanto sia i miei genitori che i miei fratelli non mi hanno mai guardato con occhi diversi, permettendomi sempre di condurre una vita cd. normale.

Infatti, ho avuto la possibilità di fare tutte le cose che facevano gli altri sin dalla prima infanzia. Ricordo con piacere i pomeriggi trascorsi, tra compiti e giochi, insieme ai compagni di scuola, così anche le feste di compleanno nei tempi dell'adolescenza, quando i genitori mi permettevano di ritagliarmi del tempo libero per le prime uscite serali. Erano anche gli anni trascorsi in oratorio, dove cercavo di approcciarsi, seppur ancora prematuramente alla fede; una dimensione che scoprivo e, scopro giorno dopo giorno, fonte da cui traggo la forza per superare i momenti difficili della mia vita.

Uno di questi l'ho vissuto nell'età adolescenziale quando, alla scoperta del proprio corpo, faceva seguito quella relativa alla sfera dei sentimenti che, in questa fase non si identificavano più soltanto negli affetti familiari e degli amici ma comprendevano anche altri tipi di emozioni che interessavano un sesso diverso dal mio.

Infatti fu proprio quando prendevo le cd. prime cotte, cominciando a guardare i ragazzi con gli occhi dell'amore che iniziavo ad interrogarmi sulla mia condizione di disabile; fu quello il momento in cui iniziavo a prendere coscienza dei miei limiti in un susseguirsi di emozioni contrastanti, in cui la gioia dei primi innamoramenti cedeva il posto alla rabbia nel vedermi "costretta " in un corpo che, nonostante gli sforzi, aveva gambe che non mi davano la possibilità di raggiungere autonomamente luoghi e persone.

Nel passare degli anni che, intanto, mi regalavano le prime grandi soddisfazioni del diploma, iniziavo ad elaborare il concetto del limite e ad interiorizzarlo nella mia esperienza di vita.

Tale processo di elaborazione, al quale faceva seguito l'esperienza universitaria, maturava tra le fatiche dello studente e la serenità per le belle amicizie ed i momenti felici trascorsi nelle aule dell'ateneo.

Diversi anni passati tra libri e serate studentesche che, in un batter d'occhio, mi hanno condotto fino al tanto atteso e sognato traguardo della laurea. L'acquisizione di quel titolo ha rappresentato per me la realizzazione di un sogno che ho inseguito sin da bambina, quando la spiccata socievolezza che mi ha sempre contraddistinto mi induceva a relazionarmi facilmente con il mondo esterno.

Una realtà questa, che per alcuni versi, mi era ancora sconosciuta, poiché sebbene fossi pienamente inserita nel contesto sociale della mia città, dove oltre a svolgere varie attività sportive, godevo anche di una rete di amicizie che scaturivano sia dall'esperienza maturata nelle associazioni di volontariato locali che da rapporti sociali di diversa natura, doveva ancora insegnarmi tanto.

Un ulteriore momento caratterizzato da emozioni negative quali rabbia, insoddisfazione, sgomento fu ed è ancora, quello post- laurea, poiché uno degli scogli più grossi da arginare è rappresentato dall'inserimento lavorativo delle persone che, come me, hanno una disabilità.

Ancora oggi, a distanza di diversi anni, dal conseguimento del titolo accademico, mi ritrovo a sperimentare l'amarezza per una mentalità ostile verso i disabili.

Dalla mia esperienza di vita ho capito, che almeno qui al meridione, tanti passi si devono fare in tal senso, non basta parlare e nascondersi dietro "false" leggi che, a tutt'oggi, stentano a trovare concreta applicazione, ma occorre una vera e propria presa di coscienza da parte di tutti per restituire a tutti i disabili la dignità che, oggi più di allora, ci viene negata!!"

\section{Discussione dei Risultati}

Lo studio ha voluto esaminare l'impatto della disabilità nel percorso universitario e lavorativo della persona portatrice di handicap. A tal scopo è stato chiesto ai soggetti di riferire come vivono quotidianamente la loro disabilità, cosa provano interiormente, come si relazionano con il contesto sociale e come questo accoglie o respinge la loro presenza. Inoltre, i partecipanti sono stati invitati ad esprimere il proprio pensiero relativo al modo in cui i rispettivi genitori vivono le difficoltà dei figli. Infine, al campione è stato dato spazio per esprimersi liberamente su qualsiasi aspetto di cui avrebbero voluto parlare. Dall'analisi qualitativa delle testimonianze raccolte è emerso che il campione preso in esame comprende trenta 
soggetti che esprimono sentimenti e reazioni differenti di fronte alla disabilità; si manifestano emozioni contrastanti ed ambivalenti e modalità diverse di reagire alle difficoltà poste dall'handicap. Tali differenze sono facilmente individuabili in emblematiche dicotomie di cluster. All'atteggiamento positivo, grintoso e desideroso di affetto e di amore si contrappongono espressioni di negatività caratterizzate dalla rabbia, dall'accusa verso il sistema e verso le figure genitoriali, a volte anche connotate da toni duri ed aggressivi, che fanno trasparire risentimento e rancore dovuti ad una non accettazione della condizione di disabilità. Tali temi sono esplicitati spesso in forma di metafore utili ad offuscare emozioni, consapevoli e non, che inevitabilmente vengono fuori nei loro racconti.

II numero ridotto del campione e la natura qualitativa dell'analisi pongono il limite della soggettività dei risultati, per cui sarebbe opportuno predisporre un test psicometrico che dia, ai dati grezzi originari, una forma standard al fine di renderli confrontabili.

La mia personale esperienza mi ha indotto ad approfondire gli aspetti emersi dalla ricerca. Questo mi ha permesso di vedere con maggiore chiarezza tutte le necessità e le lacune che una condizione di disabilità impone ed i limiti, fisici e non, a cui quotidianamente si è esposti. Pertanto ho individuato aspetti di vita quotidiana, scolastica, lavorativa e sociale che, dal punto di vista del disabile, maggiormente hanno bisogno di attenzione, al fine di migliorarne la qualità. Le tematiche che ho preso in considerazione potrebbero costituire fonte di ulteriori studi e approfondimenti scientifici.

Introdurrò innanzitutto quelli che, a mio parere, sono gli aspetti pregnanti ma lacunosi della vita domestica, estesa anche alle normali attività giornaliere fuori casa. Ritengo di fondamentale importanza il ruolo dei familiari che curano l'assistenza fisica del disabile. Data la difficoltà dei congiunti di trovare tutto il tempo necessario per assolvere tale funzione, sarebbe opportuna un'opera di sensibilizzazione che, con un incremento di ore di riabilitazione cognitiva, agisca sul nucleo familiare al fine di tramutare l'affetto in forme di dedizione ed attenzione che coinvolgano il figlio in attività quotidiane collaborative e stimolanti dal punto di vista cognitivo. Essa potrebbe concretizzarsi in una vasta gamma di forme che vanno dalle attività intellettualmente più impegnative, come la lettura di libri o la stesura di racconti, a quelle meno intense che rientrano nella sfera ludica, come l'uso di costruzioni per i disabili motori, ovviamente supportati da un aiuto effettivo, oppure semplici giochi di enigmistica ed esercizi di logica per i soggetti con ritardi mentali, sempre affiancati da figure competenti e, in ambito domestico, da genitori o fratelli, che esortino a svolgere tali attività. Allo stesso modo è auspicabile, da parte di centri diurni e case famiglia, mettere in atto il medesimo impegno, per esortare gli utenti ad una maggiore autonomia e favorire un coinvolgimento attivo nelle ADL (Activity Daily Life). Questa maggiore focalizzazione sulla terapia occupazionale porterebbe a risultati positivi tangibili nelle occupazioni quotidiane "semplici" che per determinati tipi di disabilità rappresentano una vera e propria conquista, come il gesto di bere un caffè avvicinando la tazzina autonomamente alla bocca 0 alzarsi dalla sedia e reggersi in piedi da soli per qualche secondo. Un ulteriore contributo che agevolerebbe la soluzione di alcune necessità rilevate, potrebbe essere fornito dai centri diurni, attraverso la strutturazione di spazi ricreativi in cui dare libero sfogo a passioni, attitudini e potenzialità (cucinare, dipingere, scrivere, suonare, usare il computer, ecc.) da coltivare, con l'obiettivo riabilitativo di tramutarle, in futuro, in un possibile impiego lavorativo. All'importanza della stimolazione cognitiva si affianca l'attenzione per la sfera fisica e si afferma la necessità di incrementare e garantire servizi sanitari, con i conseguenti vantaggi psicologici, come quelli fisioterapici e riabilitativi, finalizzati al miglioramento della qualità della vita quotidiana del disabile ed a sollecitare nuovi progressi ai fini di un'autonomia sempre più soddisfacente. Beneficiare della riabilitazione neuromotoria significa prendere consapevolezza di capacità fisiche che danno nuova grinta e determinazione psicologica, facendo raggiungere risultati inaspettati anche se lenti che, per tale ragione, richiedono un maggior numero di ore di esercizio che le Istituzioni potrebbero garantire, oltre che di sforzi e sacrifici fisici alimentati da coraggio e tenacia. Ritengo di primaria importanza che tali requisiti di caparbietà e fermezza ostinata siano indispensabili nei genitori, ancor prima che nel disabile stesso, per nutrire la sua perseveranza a non mollare mai!

Ho ritenuto opportuno prendere in esame il ruolo che il caregiver, assolto nella maggior parte dei casi dai genitori, assume nelle attività normali della giornata di una persona disabile. A tal proposito il Coordinamento Nazionale Famiglie di Disabili Gravi e Gravissimi ha avviato un'azione legale tesa al riconoscimento giuridico della figura del caregiver in Italia, con rivendicazioni di carattere economico-previdenziali. Poiché in Italia manca una salda rete assistenziale adeguata, l'onere di un'assistenza continua $24 \mathrm{~h}$ su $24 \mathrm{~h}$ ricade sul nucleo familiare. Questo sancisce la centralità di un ruolo non riconosciuto socialmente in maniera congrua, anche in termini economici, ma che assolve un compito primario ed essenziale. In Italia si auspica, come accade già in altri Paesi Europei, ad esempio in Germania, il riconoscimento di compensi specifici e di strumenti di tutela nei confronti di queste figure. Tale richiesta è fondata sul sacrificio di persone che dedicano la loro intera vita all'assistenza del congiunto disabile, ma anche su prove scientifiche che attestano aspettative di vita sensibilmente ridotte, dai 9 ai 17 anni, a causa dell'elevato stress quotidiano. Infatti, il premio Nobel 2009, Elizabeth Blackburn, ha mostrato insieme al suo team, come lo stress cronico, dovuto all'accudimento del familiare disabile, incrementa l'attività dell'enzima telomerasi, provocando una riduzione della vita cellulare e quindi un 
invecchiamento più rapido. Invece, nonostante i continui sforzi che, a livello legislativo, si protraggono da almeno diciotto anni, una proposta di legge per il prepensionamento dei familiari di disabili gravi giace immobile nel Parlamento Italiano registrando il record, per nulla decoroso, di proposta di legge con il più lungo iter parlamentare nella nostra Repubblica. Altrettanto utile sarebbe la possibilità di ricevere, da parte dello Stato, un'assistenza domestica continua e permanente, che alleggerisca lo sforzo fisico dei parenti e l'eventualità di richiedere una sovvenzione che, in termini finanziari, sia di sollievo per l'economia familiare e dia, al contempo, garanzia di competenza adeguata alle esigenze del disabile. Un altro bisogno che merita rilevanza è quello riguardante la cura della relazione con la badante, tenendo conto delle difficoltà che, da entrambe le parti, si manifestano nelle diversità derivanti, a volte, dall'integrazione interculturale e dall'inevitabile contrasto di pensiero determinato da una convivenza quotidiana $24 \mathrm{~h}$ su $24 \mathrm{~h}$ che, vissuta in maniera continuativa, può influire sull'intimità familiare. L'inevitabile dipendenza fisica dal caregiver 0 da un familiare, che, per ovvie necessità s'instaura, assume talvolta connotazioni negative, qualora sia limitata la libertà mentale della persona disabile.

La presenza in famiglia di una persona con disabilità fisica e/o psichica determina una ristrutturazione dell'intero sistema familiare, giacché la sofferenza derivante da una situazione così delicata investe non solo i genitori ma anche fratelli e/o sorelle sin dal momento della comunicazione della diagnosi. II modo in cui è comunicata la notizia ai fratelli, maggiori o minori, riveste un ruolo primario rispetto al futuro approccio, in età adulta, col disabile e con la stessa condizione di handicap, ma soprattutto nel rapporto con la società, con il mondo esterno alla famiglia, sia con i coetanei sia con i cosiddetti modelli proposti dai media. II modo in cui i genitori gestiscono la relazione con il figlio disabile deve essere tale da non suscitare sentimenti di trascuratezza e gelosia nei figli non disabili. II senso di abbandono risvegliato da tali sentimenti è la diretta conseguenza delle maggiori cure di cui un figlio disabile necessita. Tutto questo influenzerà le reazioni e le relazioni in età adulta dei fratelli normodotati. Pertanto ritengo che possa essere interessante predisporre un'indagine volta a rilevare il pensiero che emerge all'interno di gruppi di Auto Mutuo Aiuto costituiti da familiari e fratelli 0 sorelle di persone disabili. Potrebbe essere utile l'organizzazione di focus group che approfondiscano l'opinione che tali soggetti hanno sul rapporto tra disabilità e società.

Ho preso in considerazione anche la condizione in cui una persona disabile si ritrova nel momento in cui vengono a mancare entrambi i genitori. Tale assenza determina un senso di vuoto notevole a cui cercano di porre rimedio i già esistenti centri diurni e case famiglia, che accolgono ragazzi orfani con l'obiettivo importante di ricreare il nido familiare ed il clima domestico di cui sono stati privati. È auspicabile che queste istituzioni si presentino come una nuova e solida rete amicale su cui fare affidamento e che prendano in considerazione le eventuali difficoltà economiche dei loro utenti. In alternativa l'esigenza-diritto del disabile, soprattutto se mentale, sarebbe quella di continuare a vivere nella propria casa d'origine, magari con un contributo da parte delle istituzioni Statali e con la presenza d'assistenza di una persona garantita costantemente per tutto l'arco della giornata, al fine di alleviare la solitudine e lo smarrimento causati da un simile lutto. In una relazione di questo tipo è umano e spontaneo il dispiegarsi di sentimenti d'affetto, che sarebbe auspicabile avessero un'intensità tale da renderlo quasi un legame familiare.

Costituisce naturale desiderio per una persona disabile, maturare, in una fase della propria vita, la legittima aspirazione di aprirsi ad una relazione sentimentale, di formare una famiglia, avere dei figli e realizzarsi appieno, dunque, anche sul piano affettivo. Tale desiderio caratterizza la natura umana, ma talvolta può essere ostacolato dai familiari che non favoriscono, né sostengono come dovrebbero, il desiderio di realizzare una convivenza o un progetto matrimoniale, poiché timorosi ed insicuri loro stessi, nei confronti delle facoltà del figlio ad affrontare una vita coniugale. Questo aspetto è rintracciabile sia nel caso in cui il coniuge non disabile, nel fungere da supporto per l'altro, venga ostacolato da una famiglia che osteggia la relazione, sia quando, entrambi disabili e bisognosi di sostegno fisico, si ritrovino a scontrarsi con il disaccordo delle proprie famiglie. Riguardo a quest'aspetto sarebbe bello chiedere ai ragazzi impegnati in una relazione come la vivono, come sono influenzati dai familiari e come pensano di organizzare il loro futuro, anche in funzione delle difficoltà riscontrabili persino nell'approccio fisico.

Sulla base della mia esperienza universitaria posso asserire di aver usufruito di un soddisfacente servizio in molteplici attività didattiche e di una piacevole relazione con i tutor, che hanno contribuito adeguatamente al dispiegarsi della mia carriera accademica. Nell'Università "G. d'Annunzio" Chieti-Pescara, che ho frequentato, tutor addetti all'assistenza alla persona sono a disposizione per l'accompagnamento all'interno delle strutture del campus e presso le aule. L'assistenza didattica prevede la trascrizione degli appunti, la compilazione delle pratiche burocratiche, il reperimento di testi, cura i contatti con i docenti e l'affiancamento in sede d'esame. Da quanto emerso dalle testimonianze e in base alla mia esperienza, spesso, si riscontra una carenza del monte ore di tutorato specializzato per lezioni aggiuntive e seminari, non sempre congruo alle esigenze soggettive dello studente. Perciò potrebbe essere opportuno un provvedimento atto a ripartire i servizi riservati alla preparazione degli esami in maniera proporzionale ai bisogni didattici specifici di ciascuno studente e della relativa materia d'esame, in quanto, nel mio caso, le ore necessarie superavano, quasi sempre, quelle a mia disposizione. Riconosco la disponibilità presente nel mio Ateneo, che mi ha 
consentito, in seguito ad una mia richiesta, di avere a disposizione un'aggiunta ulteriore di ore finalizzate alla trascrizione di appunti nel corso delle lezioni previste dal piano di studi. Una problematica che ho dovuto affrontare ha interessato la stesura della tesi, per la quale l'università non prevede un apposito servizio di tutorato, quindi ho dovuto provvedere autonomamente, gravando ancora una volta sulle finanze familiari. Ė ugualmente auspicabile che sia l'università stessa a provvedere, nel caso di studenti non vedenti e ipovedenti, alla fornitura di supporti audiovisivi avanzati (braille, un sistema di scrittura e lettura a rilievo, programmi di dettatura vocale, sintetizzatori vocali), al fine ultimo di metterli in una condizione consona ad assolvere i normali impegni accademici al pari di tutti gli altri.

Inoltre, occorre menzionare l'esigenza, altrettanto fondamentale, di supporto e assistenza fisica per tutti i servizi che non riguardino l'ambito didattico ma le necessità propriamente fisiche e fisiologiche di un essere umano (mensa e servizi igienici).

Dalla considerazione di queste urgenze emerse in ambito universitario, potrebbe ipotizzarsi un'analisi delle opinioni di coloro che si occupano del tutoraggio, sia a livello organizzativo sia di chi concretamente interagisce con i disabili, per indagare il loro punto di vista.

La presenza scomoda ed ingombrante di barriere architettoniche ostacolanti è presente tanto nei confini universitari quanto al di fuori di essi. Ciò rende indispensabile la necessità di un costante supporto fisico per evitare l'intralcio del normale svolgimento di servizi utili alla quotidianità presso strutture pubbliche quali uffici postali, banche, studi medici ecc. L'impossibilità di una persona disabile di muoversi autonomamente per strada è data anche da una struttura erronea dei marciapiedi che si presentano inclinati in senso non solo longitudinale ma anche trasversale, determinando uno sbilanciamento che impedisce la discesa lineare della carrozzina, con il conseguente rischio di caduta. Inoltre, i luoghi pubblici dispongono quasi sempre di servizi igienici appositamente progettati per i disabili che, paradossalmente, data l'esagerata altezza del wc, creano spesso disagi notevoli, invece di favorire una maggiore facilità nel suo uso. L'obiettivo che si tenta affannosamente di raggiungere è l'autonomia a cui ogni disabile ambisce, ma che la realtà attuale permette ancora difficilmente di raggiungere. I limiti sono tanti ed esistono nonostante gli sforzi, evidentemente ancora non adeguati, di rimediare ad una situazione di non autosufficienza, compresa solo in parte da chi non la vive personalmente. La linearità agognata è quella che prevede un'uscita dalla propria abitazione e che, nonostante la carrozzina, renda possibile raggiungere la fermata dell'autobus, salire sul mezzo di trasporto pubblico in tempi ragionevoli e con pochi semplici step, con la naturale rapidità di una persona che non è in carrozzina. Una piattaforma automatica servirebbe a rendere agevoli la salita e la discesa dal mezzo senza arrestare il suo percorso per un tempo superiore al previsto. Un contributo ad una quotidianità autonoma è offerto dall'esistenza di sedie a rotelle ricercate che, grazie ad un'evoluta sofisticatezza, riescono a colmare le lacune del vivere giornaliero e indipendente dalla famiglia. Quello che si cerca di conquistare è la possibilità di ricevere sovvenzioni economiche statali a favore di un simile gravoso investimento che, nel caso di carrozzine elettriche per uso esterno (con un adeguato sistema di ammortizzamento, luci di posizione e di segnalazione e un motore in grado di garantire un'idonea velocità), si aggira intorno ai novemila euro. Altre tipologie altamente innovative superano i diecimila euro, sono quelle che favoriscono addirittura la possibilità di raggiungere autonomamente, grazie alla semplice pressione di un pulsante, una postura eretta consentendo pertanto di muoversi come i normodotati. Una barriera quasi del tutto annientata è rappresentata dalle scale, grazie ad innovative carrozzine molto tecnologiche che permettono di superare ogni tipologia di scalinata. Desidero sottolineare che questi ausili non sono erogati dalle Aziende Sanitarie Locali, sebbene siano di primaria utilità nel raggiungimento dell'autonomia di noi disabili.

Un diritto altrettanto importante da soddisfare, sebbene non rientri nella sfera dei bisogni basilari, è la possibilità di poter andare al mare e accedere alla spiaggia nella maniera più agevole possibile. L'esigenza di vivere il mare come chiunque altro è stata soddisfatta con successo dall'introduzione del JOB, una sedia mare che permette di entrare in acqua comodamente seduti. Ribadisco che, quello di vivere l'ambiente marino, sebbene non rientri nei diritti essenziali della persona, giacché non appartenente all'ambito delle cure mediche, s'inserisce in un quadro interpersonale e di socializzazione che desidero affrontare perché fonte di benessere psicofisico della persona.

All'interno di quest'area prettamente sociale, un'idea di ricerca potrebbe essere quella di investigare le convinzioni delle varie Istituzioni pubbliche, politiche e sanitarie, chiedendo il loro parere su tali tematiche attraverso interviste. Inoltre, sarebbe interessante esaminare la corrispondenza tra queste esigenze ed i progetti pensati per rispondere ad esse, in un'ottica di governance dei cittadini.

\section{References}

Aristotele. (1987). Poetica, a cura di Lanza D., Rizzoli, Milano.

Arulmani, G. and Nag-Arulmani, S. (2004). Career Counselling: A Handbook. New Delhi, India: Tata McGraw Hill. 
Black, M. (1954). Metaphor, Proceedings of the Aristotelian Society 55. Ristampato in Black M. (1962), Models and Metaphors. Studies in Language and Philosophy, Ithaca-Londra. Tr. It. "Metafora", in Black M. (1983).

Cortini, M. (2014). Mix-method research in applied psychology. Mediterranean Journal of Social Sciences, 5(23), 1900-1905.

Cortini, M., \& Tria, S. (2014). Triangulating qualitative and quantitative approaches for the analysis of textual materials: An introduction to T-lab. Social Science Computer Review, 32(4), 561-568.

Denzin, N. and Lincoln, Y. (1994). Handbook of Qualitative Research. Thousand Oaks, CA, Sage Publications. In Cicognani, E. (2010), Psicologia Sociale e ricerca qualitaiva, pp. 7-8, Carocci, Roma.

Dey, I. (1993). Qualitative Data Analysis, Routledge, London-New York.

Dumarsais, C. (1988). Des tropes, ou des different sens, Flammarion, Parigi.

Ferrucci, F. \& Cortini, M. (2015). Social representations and inclusive practices for disabled students in Italian Higher Education: a mix-method analysis of multiple perspectives, in T. Shakespeare (Ed) Disability Research Today. International Perspectives. Routledge, Abington, New York, pp. 185-203.

Fontanier, P. (1968). Les figures du discours, Flammarion, Parigi.

Genette, G. (1968). Introduction. In Fontanier, P. Les figures du discours. Paris: Flammarion.

Groupe $\mu$ (1970). Rhétorique général, Larousse, Parigi. Tr. it. Retorica generale. Le figure della comunicazione, Bompiani, Milano, 1976.

IRSO -International Research Ship Operators, (settembre 2000), La formazione tecnico-professionale negli USA, pp. 108-109, sez. A doc.

Lakoff G., Johnson M. (1981), Metaphors we Live by, The University of Chicago Press, Chicago-Londra. Tr. it.: Metafora e vita quotidiana, Editori Europei Associati.

Lakoff, G., Turner, M. (1989). More than Cool Reason, The University of Chicago Press, Chicago-Londra.

Lieblich, A., Tuval-Mashiach, R., Zilber, T. (1998). Narrative Research. Sage, Thousand Oaks.

Lög um framhaldsskóla, nr. 92/2008. [Law on upper secondary school], 2008.

Lög um grunnskóla, nr. 91/2008. [Law on elementary and lower secondary school], 2008.

Marshall, C. and Rossman, GB. (1995). Designing Qualitative Research, Sage, Thousand Oaks.

Super, DE. (1980). A life-span, life-space approach to career development. Journal of Vocational Behavior, 16, 282-298. 
ISSN 2039-2117 (online)

ISSN 2039-9340 (print)
Mediterranean Journal of Social Sciences MCSER Publishing, Rome-Italy
Vol 7 No 6 November 2016 